\section{PUBLICACIONES DE LA CECEL}

\author{
José Aparicio Pérez \\ Secretario General de la CECEL
}

\title{
CECEL'S PUBLICATIONS
}

RESUMEN: Se estudia la creación en 1994 de la Revista de la CECEL y su evolución y contenidos.

PALABRAS CLAVE: El Boletín de la CECEL. La revista de la CECEL. Contenidos.

A partir del año 2000, al crearse la Revista de la CECEL, tal y como relataremos seguidamente, la Revista anterior se convirtió en el Boletín de la CECEL, nombre más adecuado y que mantiene.

Actividades organizadas por el Centro en si y desde las secciones en que se organizan muchos de ellos.

La actividad principal de los Centros se dirige a la publicación de las propias investigaciones, así como las particulares de sus miembros, o bien otras hechas por investigadores y especialistas cuya materia sea de especial interés para el centro en cuestión. En este sentido, y en su conjunto, la CECEL es, sin duda, la mayor editorial hispánica y, quizás, una de las primeras de Europa, como puede observarse en la relaciòn que, en estas mismas páginas, presenta Eduardo Salazar.

Esto en cuanto número y contenido. Por lo que respecta al continente hay que decir, con la natural complacencia, que la favorable coyuntura económica de los últimos tiempos ha permitido una mejora total de la encuadernación, maquetación, papel y tipografia, alcanzándose en numerosas ocasiones lo que llamamos publicaciones de lujo, bien porque la naturaleza del contenido y su trascendencia así lo exigen, bien por la magnificencia de sus patrocinadores.

Desde la Junta de Gobierno y a partir del año 1994 se impulsó la edición de la Revista de la CECEL, especie de boletín anual, con el objetivo de informar de las actividades de los Centros y de la propia labor de la Junta de Gobierno de la Confederación a través de los informes de la Secretaría; el Editorial quedaba reservado a la Presidencia habitualmente.

Todo lo Expuesto dará idea de la intensa y extensa actividad de las partes (Centros) que constituyen el todo (la Confederación) y que enriquecen notablemente al CSIC en la que está integrada la CECEL.

El actual Boletín no ha sido ajeno a los cambios señalados, y, en 2005, a propuesta de la Secretaría de la Junta de Gobierno se acordó modificar su encuadernación con una cubierta moderna y diferente a la que, por las circunstancias expuestas, se había adoptado desde su inicio.

Corrió a cargo la nueva presentación de la Institución de Estudios Complutenses, que aceptó la modificación acordada, y que presentó la nueva, con tanto éxito que se aprobó de inmediato y la sucesiva, editada por la Real Academia de Ciencias, Bellas Letras y Nobles Artes, también la adoptó como era preceptivo y necesario.

Tras el Editorial y el Informe de Secretaría se publican las Memorias enviadas por los Centros sobre las Actividades realizadas entre las Asambleas anuales, que al celebrarse a finales de septiembre o principios de octubre vienen a coincidir con los respectivos cursos académicos y no con años naturales. 
En las Memorias se recogen resúmenes de todas las actividades realizadas por los Centros, como cursos, seminarios, congresos, simposios, jornadas, conferencias, entrevistas, nombramientos, premios dados o recibidos, asambleas, necrológicas, conciertos, exposiciones, concursos, becas, viajes culturales, homenajes, certámenes, actividades musicales, sesiones académicas en su caso, recitales poéticos, presentaciones de libros, mesas redondas, proyectos de investigación, actividades museísticas (en su caso), aperturas de curso, movimiento bibliotecario, cambios internos, etc., etcétera, y, especialmente, sus publicaciones, numerosísimas, importantes en general.

Se han publicado 13 boletines hasta el momento, uno por año, y lo han hecho los Centros siguientes.

1. Año 1994: Institut d'Estudis llerdencs.

2. Año 1995: CECEL.

3. Año 1996: Instituto de Estudios Riojanos.

4. Año 1997: Instituto de Estudios Manchegos.

5. Año 1998: Centro de Estudios Montañeses.

6. Año 1999: Real Academia de Cultura Valenciana.

7. Año 2000: Academia de Bellas Artes de Cádiz.

8. Año 2001: Instituto de Estudios Giennenses.

9. Año 2002: Instituto de Estudios Albacetenses.

10. Año 2003: Institución Fernando El Católico de Zaragoza.

11. Año 2004: Sociedad Científica el Museo Canario.

12. Año 2005: Institución de Estudios Complutenses.

13. Año 2006: Real Academia de Córdoba de Ciencias, Bellas Letras y Nobles Artes.

14. Año 2007: Instituto de Estudios Altoaragoneses. Huesca.

15. Año 2008: Real Academia "Alfonso X el Sabio". Murcia.

El año 2001, en el mes de abril se terminó de imprimir el primer número de la Revista de la CECEL, de su edición se encargó quien suscribe desde la Real Academia de Cultura Valenciana, en la que desempeñaba el cargo de Secretario Perpetuo, centro que asumió los gastos de impresión; el diseño y maquetación de la Revista también lo hizo quien suscribe, siendo aprobado por la Presidencia y la Junta de Gobierno de la CECEL.

En la presentación, escrito que encabeza los textos, dijimos lo siguiente:
La Confederación Española de Centros de Estudios Locales, nombre actual del antiguo Patronato José M. ${ }^{a}$ Cuadrado, organismo del Consejo Superior de Investigaciones Científicas, agrupa a cincuenta y seis entidades culturales de entre las de mayor solera y prestigio de España, dependientes de Ayuntamientos, Diputaciones o Comunidades Autónomas.

Consolidada su gestión, reafirmada su posición dentro del CSIC y consciente del gran papel que representa actualmente y del que está llamada a representar como eje vertebrador cultural de la España del futuro, la CECEL amplía, con su Revista, su proyección científica.

La dedica a temas hispánicos de interés común, el número 1 al estudio de los judios como minoría étnica en la España Medieval, en convivencia con moriscos y cristianos, convivencia inestable y no pacifica como algunos quieren hacer creer.

Su anterior revista queda como Boletín de asuntos internos de la Confederación, para la intercomunicación de los respectivos centros.

El nivel de la revista quiere ser el máximo y la colaboración se solicita, en primer lugar a sus centros $y$, naturalmente, a todos los demás centros nacionales o extranjeros y a investigadores que deseen contribuir con sus aportaciones a esta finalidad académica y científica.

Constituido el Consejo de Redacción que asumió la propia Junta de Gobierno, la coordinación corrió a cargo de la misma, de doña Ángela Madrid y de quien suscribe.

Decidida la temática para el número 1 , siempre de amplitud nacional, se consideró oportuno que fuera el estudio de "Minorías Étnicas en la España Medieval". Invitados los Centros a participar, con la colaboración del Instituto de Estudios Manchegos, el Instituto de Estudios Riojanos, la Institución "Fernando El Católico", el Instituto Alicantino de Cultura "Juan Gil Albert", y el Instituto de Estudios Madrileños, se pudieron recoger diez trabajos de temática judía exclusivamente, recopilada en 250 páginas.

Tras la "presentación" a cargo de quien suscribe, a la que ya hemos hecho referencia, se ofrecieron los trabajos siguientes:

Doctor F. A. Roca Traver: "Los Judíos Valencianos en la baja Edad Media (Vida, Sociedad y Cultura)"; doctor P. León Tello: "Fuero concedido por Alfonso VIII a la aljama hebrea de Haro"; doctor J. Fradejas Lebrero: "Cuatro narraciones aljamiadas"; doctor J. Hinojosa Montalvo: "Las comuni- 
dades judías valencianas a finales de la Edad Media: las vísperas del exilio"; doctor F. Miranda García: "La familia Bergerac y el crédito rural navarro en el siglo XI"; doctor A. Blasco Martínez: "Los judíos del Reino de Aragón: encuentros y desencuentros de una comunidad marginada"; doctor C. González Mínguez: "Etnia, religión y construcción nacional a propósito de la presencia judía en Álava, Guipúzcoa y Vizcaya durante la Edad Media"; doctor M. A. Motis Dolader: "La encrucijada del bautismo: el libre albedrío y los judeoconversos de la Aljama de Zaragoza (1412-18)"; doctor A. Espí Valdés: "Un cuadro de Emilio Sala: la expulsión de los judios" y, por último, el trabajo de D. C. Usillos: "Memorias de Sefarad: La herencia musical de los Sefardíes".

La Real Academia de Cultura Valenciana también asumió la edición del número 2 de la Revista, de cuya edición también nos encargamos personalmente y en cuya presentación dijimos lo siguiente.

La entrada en un nuevo milenio, el III, tras dos mil años de un devenir singular y denso, en que ha estado "escudriñando" una investigación cada vez más preparada e interesada, nos ha dado motivo para intentar poner al día el estado actual de nuestro conocimiento histórico sobre la Península Ibérica hace, aproximadamente, dos milenios, justo en el momento en que, nosotros, iniciamos nuestro cómputo temporal, nuestro calendario.

Ya lo hizo, hace veinticuatro años, don Antonio García y Bellido con su "España y los españoles hace 2.000 años" y, aunque hoy, también una sola persona pudo haberla hecho, porque las hay con suficiente preparación para ello, hemos considerado más oportuno que fuera una obra colectiva, aun a fuerza de perder la posible unidad de estilo, la uniformidad metodológica y la identidad doctrinal desde la que, cada generación y cada escuela, enfoca el devenir histórico.

Dada la naturaleza y composición de la CECEL, nada más oportuno que encargar, a los respectivos centros, el panorama histórico de su ámbito territorial y, en algún caso, al investigador que lo haya trabajado directamente aunque desde fuera del mismo, como ha ocurrido con Castilla-La Mancha, aunque con conocimiento, obviamente, del Instituto de Estudios Manchegos. Algunos centros, sin embargo, no han respondido a la llamada, por exceso de compromisos sin duda, quedando zonas en blanco, lo que es de lamentar, aunque en posteriores números intentaremos remediarlo.

La Real Academia de Cultura Valenciana aceptó, una vez más, encargarse de su edición y distribución, y el resultado del esfuerzo es el libro que ahora ofrecemos a los Centros y a la investigación.

Trece trabajos de Investigación se recibieron a cargo de trece especialistas, recogidos en cuatrocientas cuarenta y nueve páginas, fueron los siguientes:

J. M. ${ }^{a}$ Blázquez Martínez y M. ${ }^{a}$ Paz García Gelabert: "Historiografia de la España romana imperial"; A. Beltrán Martínez: "Aragón hace 2.000 años"; F. Javier García Lledó:

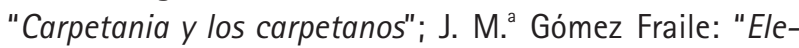
mentos para la definición del espacio geográfico de los carpetanos"; I. Ruiz Vélez: "Castilla hace 2.000 años"; C. Posac Mon: "Septem. La Ceuta de hace 2.000 años"; T. Mañanes: "El antiguo Reino de León en época romana"; R. González Femández: "La Región de Murcia hace 2.000 años"; M. Paz García Gelabert: "Paisajes y ambientes en la Meseta Sur"; M. Pastor Muñoz: "Las tierras granadinas hace 2.000 años"; M. Pastor Muñoz: "Las tierras malacitanas hace 2.000 años"; F. Costas Goberna: "Los juegos de tablero de los hispanos en los primeros siglos de nuestra Era"; J. Fradejas Lebrero: "Pompeyo Trogo y España".

El número 3 lo asumió la Institución "Fernando el Católico" de la Excma. Diputación de Zaragoza, aprovechando la celebración en Zaragoza, junto y dentro de la L Asamblea Anual de la CECEL un congreso sobre Fueros y Ordenamientos Juridicos Locales en la España Medieval, cuyas ponencias dieron contenido a la revista. En realidad, este número fue, conjuntamente, el n. ${ }^{\circ}$ 78-79/2003-2004 de la Revista de Historia "Jerónimo Zurita" de la Institución "Fernando el Católico" a la que se le puso una sobrecubierta ad hoc, que respondía al formato y estructura de las dos primeras de la CECEL, con lo que se establecian las diferencias por lo dicho.

En cuatrocientas páginas, quince investigadores nos ofrecen los trabajos siguientes:

J. M. Pérez Prendes Muñoz-Arraco: "Forum Viator. Palabras de inauguración del Congreso sobre Fueros y Ordenamientos Jurídicos Locales en la España Medieval. Zaragoza 2003"; M. Á. Ladero Quesada: "Ordenanzas locales en la Corona de Castilla"; C. Álvarez Álvarez: "Los archivos municipales: fuentes básicas para el estudio de los derechos locales en la Corona de Castilla"; G. Cavero Domínguez: "Los archivos nobiliarios y la edición y estudio de los derechos locales"; R. Morán Martín: "Horizontes matritenses del derecho de 
frontera"; L. J. Fortún Pérez de Ciriza: "Fueros locales de Navarra"; T. de Montagut Estragués: "Ordenamientos jurídicos locales catalanes"; M. de los D. Cabanes Pecourt: "Ordenamientos jurídicos locales aragoneses"; doctor E. Guinot Rodríguez: "Congreso sobre fueros y ordenamientos jurídicos locales en la España medieval"; Á. Madrid Medina: "Ordenamiento jurídico en Castilla-La Mancha: Ia presencia de las órdenes militares"; M. García Fernández: "Ordenamientos jurídicos locales andaluces (siglos XIII-XVI)"; J. L. Martín: "Ordenamientos jurídicos de la Extremadura medieval"; P. Cateura Bennàsser: "El derecho municipal como derecho del reino de Mallorca en la Edad Media"; J. R. Díaz Durana: "Ordenamientos jurídicos locales en el País Vasco y Cantabria"; C. González Mínguez: "Las asociaciones interlocales y las cartas de hermandad como generadoras de derecho".

La celebración en Alicante del Congreso sobre "Integración Ambiental y Actualización del Patrimonio Histórico", organizado por la CECEL a través del Instituto Alicantino de Cultura, "Juan Gil Albert", al celebrar éste los cincuenta años de su fundación, desde el 9 al 12 de abril de 2003 en Alicante, Orihuela y Elche, permitieron inicialmente al entonces director del Instituto, don Adrián Espi Valdés y, posteriormente, al actual director don Joaquín Santo Matas, recoger doce trabajos sobre el tema específico del Congreso $y$, con tres colaboraciones más, completar un volumen de doscientas noventa y una páginas. Tras laboriosas gestiones al final el número 4 de la Revista pudo ver la luz, ofreciéndonos los trabajos siguientes.

A. de la Banda y Vargas: "Los inicios de la recuperación del Patrimonio Histórico Español"; J. López de Uribe y Laya: "Integración ambiental del Patrimonio Histórico Español"; J. de Navascués y de Palacio: "Conservación e integración medio ambiental. Apuntes sobre la arquitectura como creadora de ambientes específicos"; I. González Tascón: "El Patrimonio Histórico en la ingeniería"; F. Díaz Pineda: "Patrimonio Histórico y medio ambiente; J. R. Romero y Femández-Pacheco: "La protección del Patrimonio Histórico documental"; A. Estévez, J. F. Baeza y C. Lancis: "Patrimonio Geológico y Geodiversidad"; M. Oliver Narbona: "Patrimonio documental, oral e inmaterial"; R. Azuar Ruiz:
"Actualización e integración del MARO en la musealización del Patrimonio Arqueológico"; J. Castaño i García: "Un ejemplo de Patrimonio Inmaterial: La Festa o Misterio de Elche"; A. Espi Valdés: "Texto de las Embajadas de la Fiesta de Moros y Cristianos de Alcoy. Patrimonio Inmaterial. El gesto y la palabra"; I. Gárate Rojas: "El Patrimonio Monumental"; OTRAS COLABORACIONES: R. M. ${ }^{a}$ Castells González: "EI camarín del monasterio de la Santa Faz de Alicante. De la reliquia sagrada a la escenificación laica"; J. A. Soler Díaz: "Arte rupestre en el MARQ"; C. Roca de Togores Muñoz: "La población de Callosa d'en Sarrià (Alicante) en el siglo XVII. Una visión a través de la antropología física".

Actualmente se prepara la edición del número 5 , a cargo del Instituto de Estudios Giennenses, con las ponencias y los trabajos presentados al Congreso del Olivo celebrado en Jaén del 27 al 29 del mes de octubre del año 2005.

El número 6 de la Revista versó, de manera monográfica, sobre la figura de la Reina Isabel la Católica, con el subtítulo genérico de "La época de Isabel la Católica", y para su coordinación y edición se ofreció la Institución Gran Duque de Alba, de Ávila, siendo presentados sus ejemplares en la Asamblea General de la CECEL celebrada en Murcia el día 27 de septiembre de 2008.

Hasta seis grandes especialistas glosaron esa época, en 164 páginas, con los siguientes trabajos: Alberto $C$. Ibáñez Pérez: "Año 1503. Ventosilla. Propiedad real"; Ángela Madrid y Medina: "Paz en la orden de Santiago y reparaciones de Isabel la Católica"; Jaime de Salazar y Acha: "Notas para el estudio de la alta nobleza castellana en el reinado de Isabel la Católica"; Esteban Sarasa Sánchez: "Fernando II de Trastámara, rey de Aragón y príncipe del Renacimiento"; M. ${ }^{a}$ Jesús Vázquez Madruga: "Ciclo Isabel la Católica. La moda femenina en Castilla"; y Fernando Villaseñor Sebastián: "El scriptorium de Isabel I. Iluminadores trabajando en casa de la Reina".

Por fin, tengo que comentar que los números dedicados a las Actas del Congreso Internacional de Ingeniería Geomática y Topográfica y sobre el Patrimonio Cultural, ya están adjudicados y en marcha.

Recibido: Octubre de 2007

Aceptado: Mayo de 2008 